\title{
THE NON-MONOTONICITY OF THE KPP SPEED WITH RESPECT TO DIFFUSION IN THE PRESENCE OF A SHEAR FLOW
}

\author{
MOHAMMAD EL SMAILY
}

(Communicated by James E. Colliander)

\begin{abstract}
In this paper, we prove via counterexamples that adding an advection term of the form Shear flow (whose streamlines are parallel to the direction of propagation) to a reaction-diffusion equation will be enough heterogeneity to spoil the increasing behavior of the KPP speed of propagation with respect to diffusion. The non-monotonicity of the speed with respect to diffusion will occur even when the reaction term and the diffusion matrices are considered homogeneous (do not depend on space variables). For the sake of completeness, we announce our results in a setting which allows domains with periodic perforations that may or may not be equal to the whole space $\mathbb{R}^{N}$.
\end{abstract}

\section{INTRODUCTION AND MOTIVATION}

Pulsating traveling fronts are particular solutions of heterogeneous reactionadvection-diffusion equations that describe propagation phenomena for combustion models, the evolution of epidemics, population dynamics and many other phenomena. This paper is dedicated to answering the question of whether the minimal speed of propagation (KPP non-linearity) is monotone with respect to the diffusion coefficient. We answer this question in the case where an advection term is present. This work is also a continuation of the paper [4, which dealt with the dependence and asymptotic behaviors of the minimal speed of propagation on the coefficients of the reaction-diffusion equation in heterogenous media.

To explain the question mathematically we start by recalling the definition of the minimal speed of propagation and the changes that happen while passing from a homogeneous to a heterogeneous setting.

1.0.1. Homogeneous framework. In the article 10 by Kolmogorov, Petrovsky and Piskunov, the setting was "homogeneous" in the following sense. The equation considered in [10] was

$$
u_{t}=\Delta u+f(u) \text { for all }(t, x) \in \mathbb{R} \times \mathbb{R}^{N},
$$

Received by the editors February 28, 2011 and, in revised form, March 9, 2011, November 11, 2011 and January 3, 2012.

2010 Mathematics Subject Classification. Primary 35K57, 92D25, 92D40, 35P15, 35P20, $76 \mathrm{~F} 10$.

Key words and phrases. Traveling fronts, reaction-diffusion, monotonicity with respect to diffusion, KPP minimal speed, shear flows, principal eigenvalue.

The author is indebted to the Center for Nonlinear Analysis and Portugal's Foundation for Science and Technology, "Fundação para a Ciência e a Tecnologia", for financial and scientific support via the Carnegie Mellon-Portugal Program. 
where $0 \leq u=u(t, x) \leq 1$ was a function defined over $\mathbb{R} \times \mathbb{R}^{N}$. The reaction term $f$ was assumed to be of KPP type. That is, $f:[0,1] \mapsto \mathbb{R}$ such that

$$
f(u)>0 \text { for all } u \in(0,1), \quad f^{\prime}(0)>0,
$$

together with the sub-linearity condition (which is usually called the "KPP condition") over the interval $[0,1]$ :

$$
\forall u \in[0,1], f(u) \leq f^{\prime}(0) u .
$$

One can see that, in equation (1.1), the diffusion and reaction terms do not depend on space and time variables and there is no advection term of the form $q(x) \cdot \nabla u$. Fixing a unitary direction $e \in \mathbb{R}^{N}$, traveling fronts in the direction of $-e$ and with a speed $c$, in such a homogeneous setting, are solutions $u(t, x)=\phi(x \cdot e+c t)=\phi(s)$ which satisfy the limiting conditions $\phi(-\infty)=0$ and $\phi(+\infty)=1$. Kolmogorov et al. [10] proved that when the reaction term is of KPP type (i.e. satisfying conditions (1.2) and (1.3) $)$, there exists a minimal speed $c^{*}=2 \sqrt{f^{\prime}(0)}$ such that a traveling front propagating in the direction of $-e$ with a speed $c$ exists if and only if $c \geq c^{*}=2 \sqrt{f^{\prime}(0)}$. If we look at the equation

$$
u_{t}=D \Delta u+f(u),
$$

for some positive constant $D$, then a simple rescaling yields that the minimal KPP speed of (1.4) is given by $c_{D}^{*}=2 \sqrt{D} \sqrt{f^{\prime}(0)}$. One can then notice that in a homogeneous setting, the minimal speed is increasing with respect to the diffusion factor (the map $D \mapsto c_{D}^{*}$ is increasing over $(0,+\infty)$ ).

1.0.2. Heterogeneous framework, notation and setting. The result of Kolmogorov et al. 10] has been generalized to media with periodic spatially dependent coefficients (see 1], 17, 20, 21] for example) and to settings with space-time dependent coefficients (see [2, 15, 16]). Here we recall some results which are very relevant to this present work and we also introduce the setting that will be considered. The equation that we will consider is of the type

$$
u_{t}(t, z)=\nabla_{z} \cdot\left(A(z) \nabla_{z} u\right)+q(z) \cdot \nabla_{z} u+f(u), \quad t \in \mathbb{R}, z \in \Omega,
$$

where the domain $\Omega:=\mathbb{R} \times \omega$ is an unbounded $C^{3}$ open connected subset of $\mathbb{R}^{N}=\mathbb{R} \times \mathbb{R}^{N-1}(N \geq 1)$. We represent each $z \in \Omega$ as

$$
z=(x, y), x \in \mathbb{R}, y \in \omega .
$$

In order to cover all possible cases, we will now give a generic description of $\omega$ which appears in $\Omega$ when the space dimension is $N>1$. The set $\omega$ is assumed to have the following structure. There exists an integer $d \in\{0, \ldots, N-1\}$ and $L_{1}>$ $0, \ldots, L_{d}>0$ so that an element $y \in \omega$ is represented by $\left(y_{1}, y_{2}\right) \in \mathbb{R}^{d} \times \mathbb{R}^{N-1-d}$ and

$$
\left\{\begin{array}{l}
\exists R \geq 0 ; \forall\left(y_{1}, y_{2}\right) \in \omega,\left|y_{2}\right| \leq R, \\
\forall\left(k_{1}, \ldots, k_{d}\right) \in L_{1} \mathbb{Z} \times \cdots \times L_{d} \mathbb{Z}, \quad \omega=\omega+\sum_{k=1}^{d} k_{i} e_{i},
\end{array}\right.
$$

where $\left(e_{i}\right)_{1 \leq i \leq N}$ is the canonical basis of $\mathbb{R}^{N}$.

- Notice that the case $d=0$ corresponds to " $\omega$ is bounded", and hence $\Omega=\mathbb{R} \times \omega$ is an infinite cylinder of section $\omega$.

- In the cases where $d \geq 1$, the set $\omega$ is unbounded. 
As particular examples of $\Omega$, one can have the whole space $\mathbb{R}^{N}=\mathbb{R} \times \mathbb{R}^{N-1}$, in which case $d=N-1$. One can also have the whole space $\mathbb{R}^{N}$ except a periodic array of holes (periodic perforations). In such a case, $d$ is also equal to $N-1$. For $N=2$, one has $d \in\{0,1\}$. The case where $d=1$ (with $N=2$ ) means that $\omega$ is unbounded connected and satisfies (1.6), and thus $\Omega=\mathbb{R}^{2}$. For $N=3, d$ can be 0,1 , or 2 . The case where $d=1$ corresponds to $\omega \subset \mathbb{R}^{2}$ bounded in one direction and unbounded in the other one. In the unbounded direction, $\omega$ has to be periodic with a period that we denoted by $L_{1}$ in the general setting above. The case where $d=2$ corresponds to $d=N-1$ and thus has been discussed above.

We mention that in all cases (even when $d=0$ ) the domain $\Omega:=\mathbb{R} \times \omega$ has a periodicity cell which we denote by $C:=[0,1] \times C_{w}$ where

$\left\{\begin{array}{l}C_{\omega}=\omega, \text { when } d=0, \\ C_{\omega}=\left\{\left(y_{1}, y_{2}\right)=\left(y_{1}^{1}, \ldots, y_{1}^{d}, y_{2}\right) \in \omega, y_{1}^{1} \in\left[0, L_{1}\right], \ldots, y_{1}^{d} \in\left[0, L_{d}\right]\right\} \text { otherwise. }\end{array}\right.$

Concerning the reaction term, we will deal only with non-linearities $f$ depending on $u$ in order to achieve the proof of non-monotonicity of the speed with respect to diffusion. Precisely, $f$ is of the homogeneous KPP type (1.2)-(1.3).

The advection term, in this paper, is assumed to be a shear flow. That is, a vector field $q(x, y)=\left(q_{1}(y), 0, \ldots, 0\right)$ of class $C^{1, \alpha}(\bar{\Omega})(\alpha>0)$. The advection is assumed to satisfy

$$
\left\{\begin{array}{l}
q_{1} \text { is }\left(L_{1}, \ldots, L_{d}\right) \text {-periodic with respect to } y(\text { whenever } d \geq 1), \\
\int_{C} q_{1}(y) d x d y=0 .
\end{array}\right.
$$

Obviously the above vector field satisfies

$$
\nabla_{x, y} \cdot q=0 \text { in } \bar{\Omega} \text { and } q \cdot \nu=0 \text { on } \partial \Omega,
$$

where $\nu$ is the outward normal on $\partial \Omega$ and is given by $\nu=\left(0, \nu_{\omega}\right)$, where $\nu_{\omega}$ is the outward normal on $\partial \omega$.

Let us now describe a wide class of diffusion coefficients for which the existence of traveling fronts and minimal speed of propagation holds according to [1. The diffusion matrix $A(x, y)=A\left(x, y_{1}, y_{2}\right)=\left(A_{i j}(x, y)\right)_{1 \leq i, j \leq N}$ is a symmetric $C^{2, \alpha}(\bar{\Omega})$ (with $\alpha>0$ ) matrix field satisfying

$$
\left\{\begin{array}{l}
A \text { is }\left(1, L_{1}, \ldots, L_{d}\right) \text {-periodic with respect to }\left(x, y_{1}\right), \\
\exists 0<\alpha_{1} \leq \alpha_{2} ; \forall(x, y) \in \Omega, \forall \xi \in \mathbb{R}^{N} \\
\alpha_{1}|\xi|^{2} \leq \sum_{1 \leq i, j \leq N} A_{i j}(x, y) \xi_{i} \xi_{j} \leq \alpha_{2}|\xi|^{2}
\end{array}\right.
$$

In the above setting where $\Omega=\mathbb{R} \times \omega$ satisfies (1.6), $q$ is of the type (1.8) and $A$ satisfies (1.9), one can rewrite (1.5) as

$$
u_{t}(t, x, y)=\nabla \cdot(A(x, y) \nabla u)+q_{1}(y) \partial_{x} u+f(u) \text { in } \mathbb{R} \times \mathbb{R} \times \omega,
$$

together with the boundary condition (in the cases where $\partial \omega \neq \emptyset$ )

$$
\nu(x, y) \cdot A \nabla_{x, y} u(t, x, y)=0,(t, x, y) \in \mathbb{R} \times \mathbb{R} \times \partial \omega .
$$

Now in this non-homogenous setting, we set $e=(1,0, \ldots, 0) \in \mathbb{R}^{N}$ as the direction of propagation. We recall from [1, 20, 21, that a pulsating traveling front 
in the direction of $-e$ that propagates with a speed $c \in \mathbb{R}$ is a classical solution $u=u(t, x, y):=\phi(x+c t, x, y)$ that connects 0 to 1 as follows:

$$
\lim _{x \rightarrow-\infty} u(t, x, y)=0 \text { and } \lim _{x \rightarrow+\infty} u(t, x, y)=1
$$

(locally in $t$ and uniformly in $(x, y)$ ) and satisfies

$$
u\left(t+\frac{1}{c}, x, y_{1}+k, y_{2}\right)=u\left(t, x+1, y_{1}, y_{2}\right)
$$

for any $k \in L_{1} \mathbb{Z} \times \cdots \times L_{d} \mathbb{Z}$. In terms of $\phi$, the latter means that $\phi$ is 1 -periodic in $x$ and $\left(L_{1}, \ldots, L_{d}\right)$-periodic in $y_{1}$. Under the assumptions (1.6), (1.8), and (1.9) on $\Omega, q$ and $A$, and having a KPP non-linearity (1.2)-(1.3), one knows from [1] that there exists a minimal speed $c^{*}:=c_{A, \Omega, q, f}^{*}(e)>0$ so that a pulsating traveling front with a speed $c$ exists if and only if $c \geq c_{\Omega, A, q, f}^{*}(e)$. The minimal speed $c^{*}$ has been expressed in terms of the coefficients of the reaction-advection-diffusion problem via a variational formula which involves eigenvalue problems in 3 and [20. This formula has been used in many works to study various asymptotic and homogenization regimes of pulsating traveling fronts (see for e.g. [4], [5], [6], [7], 8], [1], [12, 14], 18, 22]). It has also been generalized to equations with time-space dependent coefficients (almost periodic in time and periodic in space) in Shen [19].

\section{ThE NON-MONOTONICITY OF THE MINIMAL SPEED}

\section{WITH RESPECT TO DIFFUSION (IN THE PRESENCE OF AN ADVECTION FIELD)}

Berestycki, Hamel, and Nadirashvili 3. proved (in part 2 of Theorem 1.10) that, having any periodic domain $\Omega \subseteq \mathbb{R}^{N}, q \equiv 0$ and a constant growth rate $f_{u}^{\prime}(x, y, 0)$ (which holds in the case $f=f(u)$ ), the map $\beta \mapsto c_{\Omega, \beta A, 0, f}^{*}(e)$ is increasing in $\beta>0$. Nadin [14 has proved in Theorem 2.5 that the same result still holds even when $f_{u}^{\prime}(x, y, 0)$ depends on the spatial variables $(x, y)$. However, we notice in results of both [3, 14] the absence of an advection term.

Owing to Theorem 1.1 in [3], the map $A \mapsto c_{\Omega, A, q, f}^{*}(e)$, where $A$ varies in the ordered family 1$]$ of positive definite matrices satisfying (1.9), is well defined (provided that $\Omega, q$ and $f$ satisfy (1.6), (1.8) and (1.2)-(1.3)). Having those results in [3], 4 ] and [14, there naturally arises the following question.

Question. Do we still have the increasing behaviour of the minimal speed with respect to the diffusion factor $\beta$ in the presence of an advection, even if the nonlinearity is homogenous? This question can be rephrased as: does the speed remain monotonic over the family of diffusions proportional to a prefixed diffusion $A$ in the presence of an advection? We prove in this present work that this is not true in the case where $f$ is fixed and the advection $q$ is large enough. Moreover, we give a counterexample to the monotonicity of the speed with respect to diffusion in the case where $A$ and $B$ are two non-proportional positive definite matrices satisfying (1.9) and the advection field is arbitrarily chosen (it does not need to be large as in the case of proportional diffusions).

Before starting to answer the above question, we mention that, in the KPP setting, Majda and Souganidis [13] have discussed in detail the role of advection and the possible failure of Huygens' principle. The main point of [13] is that the

\footnotetext{
${ }^{1}$ We say that $A=A(x, y) \leq B=B(x, y)$ if and only if for each $(x, y) \in \Omega$ and for each $\xi \in \mathbb{R}^{N}$, we have $\xi \cdot A(x, y) \xi \leq \xi \cdot B(x, y) \xi$. Also, we say that $A<B$ if and only if for each $(x, y) \in \Omega$ and for each $\xi \in \mathbb{R}^{N} \backslash\{0\}$, we have $\xi \cdot A(x, y) \xi<\xi \cdot B(x, y) \xi$.
} 
corresponding large-scale limit (corresponding to $\varepsilon \rightarrow 0$ ) in the wave processes of KPP-type reaction-diffusion equations does not generally evolve according to the classical Huygens principle. It is also worth mentioning that Theorem 4.1 of Kiselev and Zlatoš 9 gave, in the case of a combustion non-linearity ( $f$ having an ignition temperature), a relation between the amplitude and the period of the advection field $q$ so that $q$ acts as quenching (extinction happens).

As a matter of fact, in our setting, the presence of a shear flow will change the monotonic behavior of the minimal speed with respect to diffusion. We prove in Subsection 2.1 that the answer is negative in general even when the diffusion matrices $A$ and $B$ are proportional to the identity matrix and the non-linearity is homogenous. We give a counterexample when the advection is large (up to a scaling, this gives a counterexample to the monotonicity of the speed with respect to diffusion with a prefixed term and a small reaction). In Subsection 2.2, we prove that for a fixed non-zero shear flow and a fixed reaction, the answer to the second part of the question is negative in general for matrices $A \leq B$ which are not equal up to a positive scalar. We mention that the second result cannot be seen as a trivial consequence of the first one by using the argument that "a small perturbation of the strict inequality (2.2) remains a strict inequality and one can then perturb the diffusion matrices" (see more precise details at the beginning of Subsection 2.2). Lastly, we mention that our counterexamples apply when the diffusion matrices do not depend on the variable $x$. One knows that in such a case the traveling fronts of the reaction-advection-diffusion problem (1.10) will have the form $\phi(x+c t, y)$ and will be invariant with respect to the frame moving in the direction $-x$ (or $-e)$. Moreover, we know that in a homogenous setting the speed is monotone with respect to diffusion factors (see Section 1.0.1). Thus, the closer we are to a homogeneous setting, the harder it is to prove non-monotonicity of the KPP speed with respect to diffusion. Indeed, the setting where we construct our counterexamples can be taken very close to the homogeneous one, with only one heterogeneity coming from the advection. This gives sharpness to our results.

2.1. A counterexample in the case of proportional diffusions. In this subsection, we will show a reaction-advection-diffusion problem whose diffusion matrix varies in the subfamily of positive definite matrices $P D_{I d}=\{\beta I d, \beta>0\}$ (where $I d$ stands for the $N \times N$ identity matrix), while a shear flow will make Part 2 of Theorem 1.10 in [3] valid no longer, even if the non-linearity $f$ is taken to be homogeneous. Let us first announce the following result.

Theorem 2.1. Let $e=(1,0, \ldots, 0) \in \mathbb{R}^{N}, \Omega=\mathbb{R} \times \omega \subseteq \mathbb{R}^{N}$ satisfying (1.6) where $\omega$ may or may not be bounded. Assume that the non-linearity $f=f(u)$ is a homogenous "KPP" non-linearity satisfying (1.2)-(1.3), and let us start with a shear flow $q(x, y)=\left(q_{1}(y), 0, \ldots, 0\right)$ defined over $\Omega$ such that $q_{1} \neq \equiv 0$ (satisfying (1.8) when $d \geq 1$ ) and so that

$$
0<2 \sqrt{f^{\prime}(0)}+\delta<\max _{y \in \bar{\omega}}\left(q_{1}(y)\right)-\delta, \text { for some } \delta>0 .
$$

Then, there exists $M_{1}:=M_{1}(\delta)>0$ for which there corresponds $0<\varepsilon_{1}:=$ $\varepsilon_{1}\left(\delta, M_{1}\right)<M_{1}$ such that

$$
\forall 0<\varepsilon \leq \varepsilon_{1}<M_{1}, c_{\Omega, \varepsilon I d, \sqrt{M_{1}} q, f}^{*}(e)>c_{\Omega, M_{1} I d, \sqrt{M_{1}} q, f}^{*}(e) .
$$


Proof. Before going into further detail, we recall that the variational formula of the KPP minimal speed (see [3] for example) yields the continuity of $(\kappa, \rho, \mu) \mapsto$ $c_{\Omega, \kappa A, \rho q, \mu f}^{*}(e)$ with respect to $\kappa>0, \mu>0$ and $\rho \in \mathbb{R}$. This continuity with respect to the factors of the reaction, diffusion and advection will be useful in constructing our proof. Due to the presence of several parameters in the problem, we proceed in simple steps.

Step 1. In the author's work [4, an asymptotic regime for the speed $c^{*}$ within large diffusions $M A$ together with advection fields of the form $M^{\gamma} q$ was proved (where $M \rightarrow+\infty)$. The exponent $\gamma$ was allowed to be any number in the interval $(0,1 / 2]$. Precisely, Theorem 4.1 of [4], with $\gamma=1 / 2$, yields that

$$
\lim _{M \rightarrow+\infty} \frac{c_{\Omega, M I d, \sqrt{M} q, f}^{*}(e)}{\sqrt{M}}=2 \sqrt{f^{\prime}(0)} .
$$

Using the above together with the continuity of $c^{*}$ with respect to $M$, there then exists $M_{0}:=M_{0}(\delta)>0$ such that

$$
\forall M \geq M_{0}(\delta), 0<c_{\Omega, M I d, \sqrt{M} q, f}^{*}(e)<\sqrt{M}\left(2 \sqrt{f^{\prime}(0)}+\delta\right) .
$$

Step 2. We fix $M_{1}>\max \left(1, M_{0}(\delta)\right)$. Then,

$$
0<c_{\Omega, M_{1} I d, \sqrt{M_{1}} q, f}^{*}(e)<\sqrt{M_{1}}\left(2 \sqrt{f^{\prime}(0)}+\delta\right) .
$$

Step 3. For the fixed number $M_{1}$, we also have $\sqrt{M_{1}} q$ in the form of shear flow. We now look at the effect of small diffusion while $\sqrt{M_{1}} q$ is considered as an advection field. Indeed, Theorem 3.3 of El Smaily [4 yields that

$$
\lim _{\varepsilon \rightarrow 0^{+}} c_{\Omega, \varepsilon I d, \sqrt{M_{1}}}^{*} q, f(e)=\max _{y \in \bar{\omega}}\left(\sqrt{M_{1}} q_{1}(y)\right)=\sqrt{M_{1}} \max _{y \in \bar{\omega}}\left(q_{1}(y)\right) .
$$

Consequently, there exists $\varepsilon_{1}=\varepsilon_{1}\left(\delta, M_{1}\right) \in\left(0, M_{1}\right)$ (we can choose $\varepsilon_{1}$ as small as we like) such that

$$
\begin{aligned}
\forall 0<\varepsilon \leq \varepsilon_{1}, c_{\Omega, \varepsilon I d, \sqrt{M_{1}} q, f}^{*}(e) & >\sqrt{M_{1}} \max _{y \in \bar{\omega}}\left(q_{1}(y)\right)-\delta \\
& >\sqrt{M_{1}}\left[\max _{y \in \bar{\omega}}\left(q_{1}(y)\right)-\delta\right]>0 .
\end{aligned}
$$

Step 4. Owing to (2.1), (2.3) and (2.4), one then gets

$$
\forall 0<\varepsilon \leq \varepsilon_{1}, c_{\Omega, \varepsilon I d, \sqrt{M_{1}} q, f}^{*}(e)>c_{\Omega, M_{1} I d, \sqrt{M_{1}} q, f}^{*}(e),
$$

and this completes the proof.

2.2. Case of non-proportional diffusions (another counterexample). In Subsection 2.1. we saw that a prefixed advection field was multiplied by a large enough constant $M_{1}$ in order to spoil the increasing behavior of the minimal speed with respect to diffusion matrices which are proportional to the identity matrix. A simple scaling of the reaction-advection-diffusion equations that correspond to Theorem 2.1 implies that, under the same notation,

$$
c_{\Omega,\left(\varepsilon / \sqrt{M_{1}}\right) I d, q, f / \sqrt{M_{1}}}^{*}(e)>c_{\Omega, \sqrt{M_{1}}}^{*} I d, q, f / \sqrt{M_{1}}(e),
$$

and by continuity of the minimal speed with respect to diffusion coefficients, one can then find non-proportional matrices $A$ and $B$ (in the neighborhood of $\left(\varepsilon / \sqrt{M_{1}}\right) I d$ 
and $\sqrt{M_{1}} I d$ respectively) that satisfy the general assumptions of Section 1 and such that $A<B$ and

$$
c_{\Omega, A, q, f / \sqrt{M_{1}}}^{*}(e)>c_{\Omega, B, q, f / \sqrt{M_{1}}}^{*}(e) .
$$

This can be summarized as a perturbation argument applied to a strict inequality. However, we notice that the latter inequality corresponds to reaction terms which are small. The importance of the result we give in this subsection is that it leads to the construction of a counterexample to the monotonicity of the speed with respect to diffusion while $q$ and $f$ are prefixed (neither small nor large). It turns out that the counterexample given in Theorem 2.3 concerns diffusion matrices which are non-proportional by construction and not by a perturbation argument of the result of the previous section.

Notation 2.2. For each $b>0$, let $A_{b}$ denote the $N \times N$ symmetric positive definite matrix having the form

$$
A_{b}=\left(\begin{array}{ccccc}
1 & 0 & \ldots & \ldots & 0 \\
0 & b & \ddots & & \vdots \\
\vdots & \ddots & \ddots & \ddots & \vdots \\
\vdots & & \ddots & \ddots & 0 \\
0 & \ldots & \ldots & 0 & b
\end{array}\right) .
$$

Theorem 2.3. Let $e=(1,0, \ldots, 0) \in \mathbb{R}^{N}, \Omega=\mathbb{R} \times \omega \subseteq \mathbb{R}^{N}$, where $\omega$ can be either bounded or unbounded satisfying (1.6), and let $q=\left(q_{1}(y), 0, \ldots, 0\right)$ be a shear flow satisfying (1.8), where $q_{1} \not \equiv 0$ on $\bar{\omega}$. Assume that the non-linearity satisfies (1.2)(1.3). For each $b>0$, consider the reaction-advection-diffusion problem

$$
\left\{\begin{aligned}
u_{t}(t, x, y) & =\nabla \cdot\left(A_{b} \nabla u\right)+q_{1}(y) \partial_{x} u+f(u) \\
& =\partial_{x x} u+b \Delta_{y} u+q_{1}(y) \partial_{x} u+f(u) \text { in } \mathbb{R} \times \Omega, \\
\nu(x, y) \cdot A_{b} \nabla_{x, y} u & =\nu_{\omega}(y) \cdot \nabla_{y} u(t, x, y)=0 \text { for }(t, x, y) \in \mathbb{R} \times \mathbb{R} \times \partial \omega,
\end{aligned}\right.
$$

where $A_{b}$ is the matrix introduced in Notation 2.2. Then,

$$
\begin{gathered}
\lim _{b \rightarrow+\infty} c_{\Omega, A_{b}, q, f}^{*}(e)=2 \sqrt{f^{\prime}(0)}, \\
\lim _{b \rightarrow 0^{+}} c_{\Omega, A_{b}, q, f}^{*}(e)=\max _{\bar{\omega}}\left(q_{1}(y)\right)+2 \sqrt{f^{\prime}(0)} .
\end{gathered}
$$

Proof. Consider the following change of variables:

$$
\forall(t, x, y) \in \mathbb{R} \times \mathbb{R} \times \omega, v(t, x, y)=u\left(t, \frac{x}{\sqrt{b}}, y\right) .
$$

Thus, $\forall(t, x, y) \in \mathbb{R} \times \mathbb{R} \times \omega$,

$$
\begin{gathered}
v_{t}(t, x, y)=u_{t}\left(t, \frac{x}{\sqrt{b}}, y\right), \partial_{x} v(t, x, y)=\frac{1}{\sqrt{b}} \partial_{x} u\left(t, \frac{x}{\sqrt{b}}, y\right), \\
\partial_{x x} v(t, x, y)=\frac{1}{b} \partial_{x x} u\left(t, \frac{x}{\sqrt{b}}, y\right) \text { and } \Delta_{y} v(t, x, y)=\Delta_{y} u\left(t, \frac{x}{\sqrt{b}}, y\right) .
\end{gathered}
$$

Owing to the invariance of $\Omega$ in the $x$-direction, we have the boundary condition

$$
\forall(t, x, y) \in \mathbb{R} \times \partial \Omega, \quad \nu(x, y) \cdot \nabla_{x, y} v(t, x, y)=0 .
$$


Consequently, problem (2.5) is equivalent to the problem

$$
\left\{\begin{aligned}
v_{t}(t, x, y) & =b \partial_{x x} v+b \Delta_{y} v+\sqrt{b} q_{1}(y) \partial_{x} v(t, x, y)+f(v) \\
& =b \Delta_{x, y} v+\sqrt{b} q_{1}(y) \partial_{x} v+f(v) \text { in } \mathbb{R} \times \mathbb{R} \times \omega, \\
\nu(x, y) \cdot \nabla_{x, y} v(t, x, y) & =0 \text { for }(t, x, y) \in \mathbb{R} \times \mathbb{R} \times \partial \omega
\end{aligned}\right.
$$

The minimal speed of problem (2.8) exists in this setting (according to Theorem 1.2 in [1]) and is denoted here by $c_{\Omega, b I d, \sqrt{b} q, f}^{*}(e)$, where $I d$ stands for the identity $N \times N$ matrix. As in the proof of Theorem 2.1 we apply Theorem 4.1 of [4] with $\gamma=1 / 2$ to conclude that

$$
\lim _{b \rightarrow+\infty} \frac{c_{\Omega, b I d, \sqrt{b}, f}^{*}(e)}{\sqrt{b}}=2 \sqrt{f^{\prime}(0)} .
$$

On the other hand, looking at problems (2.5) and (2.8) and the relation between $u$ and $v$ and owing to the minimality (uniqueness) of the threshold $c^{*}$, one knows that the corresponding minimal speeds have the following relation:

$$
\forall b>0, c_{\Omega, b I d, \sqrt{b} q, f}^{*}(e)=\sqrt{b} c_{\Omega, A_{b}, q, f}^{*}(e) .
$$

Together with (2.9), we obtain that

$$
\lim _{b \rightarrow+\infty} c_{\Omega, A_{b}, q, f}^{*}(e)=2 \sqrt{f^{\prime}(0)} .
$$

Proof of (2.7). For the limit as $b \rightarrow 0^{+}$, we know from the rescaling formula (2.10) that proving (2.7) is equivalent to proving that

$$
\lim _{b \rightarrow 0^{+}} \frac{c_{\Omega, b I d, \sqrt{b} q, f}^{*}(e)}{\sqrt{b}}=\max _{\bar{\omega}}\left(q_{1}(y)\right)+2 \sqrt{f^{\prime}(0)} .
$$

Indeed, one can apply similar techniques to those used in the proof of Theorem 3.3 of [4] ( $\zeta$ replaced by $\left.f^{\prime}(0)\right)$ to prove this. For the sake of completeness, we do the proof here in detail. One knows that $c_{\Omega, b I d, \sqrt{b} q, f}^{*}(e)$ is given by the variational formula of $[3]$ :

$$
\forall b>0, c_{\Omega, b I d, \sqrt{b} q, f}^{*}(e)=\min _{\lambda>0} \frac{k(\lambda)}{\lambda},
$$

where $k(\lambda)$ is the principal eigenvalue of the following problem:

$$
\begin{aligned}
L_{\Omega, e, b I d, q, f, \lambda} \psi= & b \Delta \psi+2 b \lambda e \cdot \nabla \psi+\sqrt{b} q_{1}(y) \partial_{x} \psi \\
& +\left[b \lambda^{2}+\lambda \sqrt{b} q_{1}(y)+f^{\prime}(0)\right] \psi \text { in } \Omega=\mathbb{R} \times \omega,
\end{aligned}
$$

with the boundary conditions $\nu \cdot \nabla \psi=\lambda \nu \cdot e \psi$ on $\partial \Omega$. The eigenfunction $\psi=$ $\psi\left(x, y_{1}, y_{2}\right)$ is positive in $\bar{\Omega}, 1$-periodic in $x,\left(L_{1}, \ldots, L_{d}\right)$-periodic in $y_{1}$, and unique up to multiplication by a non-zero constant. Now, having the coefficient $q_{1}$ independent of $x$, this yields that one can choose $\psi$ independent of $x$. Hence, the elliptic operator $L_{\Omega, e, b I d, \sqrt{b} q, f, \lambda}$ can be reduced to the symmetric operator

$$
L_{\Omega, e, b I d, \sqrt{b} q, f, \lambda} \psi=b \Delta \psi+\left[b \lambda^{2}+\lambda \sqrt{b} q_{1}(y)+f^{\prime}(0)\right] \psi .
$$


Consequently, we have the following Rayleigh quotient over $H^{1}\left(C_{\omega}\right)$, where $C_{\omega}$ is the periodicity cell of $\omega$ introduced in Section 1 above:

$(2.14)$

$$
\begin{aligned}
& \forall \lambda>0, \forall b>0, \\
& -k(\lambda)=\min _{\varphi \in H^{1}\left(C_{\omega}\right) \backslash\{0\}} \frac{b \int_{C_{\omega}}|\nabla \varphi|^{2} d y-\lambda \sqrt{b} \int_{C_{\omega}} q_{1}(y) \varphi^{2}-\int_{C_{\omega}}\left[\lambda^{2} b+f^{\prime}(0)\right] \varphi^{2}(y) d y}{\int_{C_{\omega}} \varphi^{2}(y) d y} .
\end{aligned}
$$

Formula (2.14) leads directly to the upper bound

$$
\forall \lambda>0, \forall b>0, \frac{k(\lambda)}{\lambda \sqrt{b}} \leq \max _{y \in \bar{\omega}}\left(q_{1}(y)\right)+\lambda \sqrt{b}+\frac{f^{\prime}(0)}{\lambda \sqrt{b}} .
$$

Testing the right hand side at $\lambda_{0}=\sqrt{\frac{f^{\prime}(0)}{b}}$, one then concludes that

$$
\forall b>0, \min _{\lambda>0} \frac{k(\lambda)}{\lambda \sqrt{b}} \leq \max _{y \in \bar{\omega}}\left(q_{1}(y)\right)+2 \sqrt{f^{\prime}(0)}
$$

and hence

$$
\limsup _{b \rightarrow 0^{+}} \frac{c_{\Omega, b I d, \sqrt{b} q, f}^{*}(e)}{\sqrt{b}} \leq \max _{y \in \bar{\omega}}\left(q_{1}(y)\right)+2 \sqrt{f^{\prime}(0)} .
$$

For the sharp lower bound of the liminf as $b \rightarrow 0^{+}$, we proceed as follows. Let $y_{0}$ be a point of $C_{\omega} \subseteq \bar{\omega}$ where the periodic function $q_{1}(y)$ attains its positive maximum (we recall here that $q_{1} \not \equiv 0, q_{1}$ is periodic in $y$ and has a zero average over $C_{w}$, and thus the point $y_{0}$ is in the interior of the cell $C_{\omega}$ ). Then, by the definition of a maximum point of a continuous function, for each $0<\delta<q_{1}\left(y_{0}\right)$, there exists a neighborhood $U_{\delta} \subset C_{\omega}$ of $y_{0}$ such that

$$
\forall y \in U_{\delta}, q_{1}(y) \geq q_{1}\left(y_{0}\right)-\delta \geq 0 .
$$

Now we test the Rayleigh quotient against a smooth function $\phi(y)$ compactly supported in $U_{\delta}$ and normalized by $\int_{C_{\omega}} \phi^{2} d y=1$ to get (after switching from $-k(\lambda)$ to $+k(\lambda))$

$$
\frac{k(\lambda)}{\lambda} \geq \lambda b+\frac{1}{\lambda} \beta(b)+\sqrt{b}\left(q_{1}\left(y_{0}\right)-\delta\right),
$$

where $\beta(b)=f^{\prime}(0)-b \int_{U_{\delta}}|\nabla \phi|^{2}$. We notice that $\beta(b)>0$ for $0<b<b_{0}:=$ $\frac{f^{\prime}(0)}{\int_{U_{\delta}}|\nabla \phi|^{2}}$. The function $\lambda \mapsto \lambda b+\frac{1}{\lambda} \beta(b)$ attains its minimum over $(0,+\infty)$ at $\lambda_{*}=\sqrt{\frac{\beta(b)}{b}}$, and the value of this minimum is $2 \sqrt{b} \sqrt{\beta(b)}$. Referring to (2.16)), one then gets

$$
\forall 0<b<b_{0}, \forall \lambda>0, \frac{k(\lambda)}{\lambda} \geq 2 \sqrt{b} \sqrt{\beta(b)}+\sqrt{b}\left(q_{1}\left(y_{0}\right)-\delta\right) .
$$

The right hand side of the last inequality is independent of $\lambda$. Thus, the variational formula (2.12) yields that

$$
\forall 0<b<b_{0}, \frac{c_{\Omega, b I d, \sqrt{b} q, f}^{*}(e)}{\sqrt{b}} \geq 2 \sqrt{\beta(b)}+\left(q_{1}\left(y_{0}\right)-\delta\right),
$$


for all $0<\delta<q_{1}\left(y_{0}\right)$. Thanks to the non-dependence of $U_{\delta}$ on $b$, we pass to the limit as $b \rightarrow 0^{+}$to get

$$
\forall 0<\delta<q_{1}\left(y_{0}\right), \liminf _{b \rightarrow 0^{+}} \frac{c_{\Omega, b I d, \sqrt{b} q, f}^{*}(e)}{\sqrt{b}} \geq 2 \sqrt{f^{\prime}(0)}+\left(q_{1}\left(y_{0}\right)-\delta\right) .
$$

This is enough to conclude that

$$
\liminf _{b \rightarrow 0^{+}} \frac{c_{\Omega, b I d, \sqrt{b} q, f}^{*}(e)}{\sqrt{b}} \geq 2 \sqrt{f^{\prime}(0)}+\left(q_{1}\left(y_{0}\right)\right)
$$

and, together with (2.15), finishes the proof of (2.7).

A counterexample as an application of Theorem 2.3 . Let $e=(1,0, \ldots, 0)$ and $\Omega=$ $\mathbb{R} \times \omega$ (in particular, $\Omega$ can be taken as the whole space $\mathbb{R}^{N}$ ). Choose $f=f(u)$ and $q=\left(q_{1}(y), 0, \cdots, 0\right)$ with $\int_{C} q_{1}(y) d y=0$ and $q_{1} \not \equiv 0$. Thus there exists $\delta>0$ such that

$$
2 \sqrt{f^{\prime}(0)}+\delta<\max _{y \in \bar{\omega}}\left(q_{1}(y)\right)+2 \sqrt{f^{\prime}(0)}-\delta .
$$

It follows from Theorem 2.3 above and from the continuity of the speed with respect to the diffusion factor (variational formula of [3]) that there exist $\varepsilon_{0}>0$ and $M_{0}>0$ such that

$$
\begin{gathered}
\forall 0<\varepsilon \leq \varepsilon_{0}, \quad c_{\Omega, A_{\varepsilon}, q, f}^{*}(e)>\max _{y \in \bar{\omega}}\left(q_{1}(y)\right)+2 \sqrt{f^{\prime}(0)}-\delta \quad \text { and } \\
\forall M \geq M_{0}>0, \quad c_{\Omega, A_{M}, q, f}^{*}(e)<2 \sqrt{f^{\prime}(0)}+\delta .
\end{gathered}
$$

Consequently, choosing $\varepsilon$ small enough and $M$ large enough, it follows that $A_{M} \geq A_{\varepsilon}$ in the sense of order relation on positive definite matrices; however,

$$
c_{\Omega, A_{M}, q, f}^{*}(e)<c_{\Omega, A_{\varepsilon}, q, f}^{*}(e) .
$$

Therefore the answer to the second part of the question is negative in general even when the advection is a fixed shear flow and the non-linearity $f$ is fixed and homogenous.

\section{ACKNOWLEDGMENTS}

The author would like to thank Professor François Hamel for bringing this problem to his attention during his Ph.D. work under Professor Hamel's supervision. The author would also like to thank him for his valuable, fruitful and precise suggestions while reading a preprint of this work.

\section{REFERENCES}

[1] H. Berestycki, F. Hamel, Front propagation in periodic excitable media, Comm. Pure Appl. Math. 55 (2002), pp. 949-1032. MR.1900178 (2003d:35139)

[2] H. Berestycki, F. Hamel, G. Nadin, Asymptotic spreading in heterogeneous diffusive excitable media, Journal of Functional Analysis 255 (2008), pp. 2146-2189. MR2473253 (2009k:35136)

[3] H. Berestycki, F. Hamel, N. Nadirashvili, The Speed of Propagation for KPP Type Problems (Periodic Framework), J. Eur. Math. Soc. 7 (2005), pp. 173-213. MR2127993 (2005k:35186)

[4] M. El Smaily, Pulsating travelling fronts: Asymptotics and homogenization regimes, European J. Appl. Math. 19 (2008), pp. 393-434. MR2431698 (2009j:35147)

[5] M. El Smaily, F. Hamel, L. Roques, Homogenization and influence of fragmentation in a biological invasion model, Discrete Contin. Dyn. Syst. 25 (2009), pp. 321-342. MR2525180 (2010j:35040) 
[6] M. El Smaily, S. Kirsch, The speed of propagation for KPP reaction-diffusion equations within large drift, Advances in Differential Equations 16 (2011), Numbers 3-4, pp. 361-400. MR.2767082(2012a:35154)

[7] M. El Smaily, The homogenized equation of a heterogenous reaction-diffusion model involving pulsating traveling fronts, Communications in Mathematical Sciences (CMS) 9 (2011), No. 4, pp. 1113-1128. MR2901819

[8] M. El Smaily, F. Hamel, R. Huang, Two dimensional curved fronts in a periodic shear flow, Nonlinear Analysis: Theory, Methods \& Applications 74 (2011), pp. 6469-6486. MR2833431

[9] A. Kiselev, A. Zlatoš, Quenching of combustion by shear flows, Duke Math. J. 132 (2006), pp. 49-72. MR2219254(2007b:35199)

[10] A. N. Kolmogorov, I. G. Petrovsky, N. S. Piskunov, Étude de l'équation de la diffusion avec croissance de la quantité de matiére et son application a un probléme biologique, Bulletin Université d'Etat à Moscou (Bjul. Moskowskogo Gos. Univ.), Série internationale A1 (1937), pp. $1-26$.

[11] B. Li, H. F. Weinberger, L.A. Lewis, Spreading speeds as slowest wave speeds for cooperative systems, Math. Biosci. 196 (2005), no. 1, pp. 82-98. MR2156610 (2006g:92069)

[12] Xing Liang, Xiaotao Lin, Hiroshi Matano, A variational problem associated with the minimal speed of travelling waves for spatially periodic reaction-diffusion equations, Trans. Amer. Math. Soc. 362 (2010), pp. 5605-5633. MR2661490(2011d:35243)

[13] A. Majda, P. Souganidis, Large scale front dynamics for turbulent reaction-diffusion equations with separated velocity scales, Nonlinearity 7 (1994), no. 1, pp. 1-30. MR1260130 (95e:35180)

[14] G. Nadin, Some dependence results between the spreading speed and the coefficients of the space-time periodic Fisher-KPP equation, European J. Appl. Math. 22 (2011), no. 2, pp. 169185. MR2774781 (2012c:35214)

[15] G. Nadin, Pulsating traveling fronts in space-time periodic media, C.R. Acad. Sci. Paris I 346 (2008), pp. 951-956. MR2449634 (2009i:35182)

[16] G. Nadin, Reaction-diffusion equations in space-time periodic media, C.R. Acad. Sci. Paris I 345 (2007), pp. 489-493. MR2375108 (2008j:35102)

[17] N. Shigesada, K. Kawasaki, E. Teramoto, Spatial segregation of interacting species, J. Theoret. Biol. 79 (1979), pp. 83-99. MR540951 (80e:92038)

[18] L. Ryzhik, A. Zlatoš, KPP pulsating front speed-up by flows, Comm. Math. Sci. 5 (2007), pp. 575-593. MR2352332(2008h:35200)

[19] W. Shen, Variational principle for spreading speeds and generalized propagating speeds in time almost periodic and space periodic KPP models, Trans. Amer. Math. Soc. 362 (2010), no. 10, pp. 5125-5168. MR2657675(2011h:35162)

[20] H. F. Weinberger, On spreading speeds and traveling waves for growth and migration models in a periodic habitat, J. Math. Biol. 45 (2002), no. 6, pp. 511-548. MR 1943224 (2004b:92043a)

[21] J. X. Xin, Front propagation in heterogeneous media, SIAM Review 42 (2000), pp. 161-230. MR.1778352 (2001i:35184)

[22] A. Zlatoš, Sharp asymptotics for KPP pulsating front speed-up and diffusion enhancement by flows, Arch. Ration. Mech. Anal. 195 (2010), pp. 441-453. MR2592283 (2011b:35248)

Department of Mathematical Sciences and Center for Nonlinear Analysis, Carnegie Mellon University, Wean Hall, 5000 Forbes Avenue, Pittsburgh, Pennsylvania 15213 and - Departamento de Matemática, Instituto Superior Técnico, Av. Rovisco Pais 1049-001 Lisboa, Portugal

Current address: Department of Mathematics, University of Toronto, 40 St. George Street, Room 6290, Toronto, ON, M5S 2E4, Canada

E-mail address: elsmaily@math.toronto.edu 